International Journal of Modern Physics A

(C) World Scientific Publishing Company

\title{
New Signatures For Top In Hadron Collider
}

\author{
Tao Han * \\ Department of Physics, University of Wisconsin, Madison, WI 53706, \\ and Institute of Theoretical Physics, Academia Sinica, Beijing 100080, China \\ German Valencia †and Yili Wang $\ddagger$ \\ Department of Physics and Astronomy, Iowa State University \\ Ames, Iowa 50010
}

Received (27 10 2004)

\begin{abstract}
We study the signatures for new $\mathrm{TeV}$ resonances that couple to top or bottom quarks both at the Tevatron Run II and at the LHC. We find that it is possible to study these resonances when they are produced in association with a pair of heavy quarks or in association with a single top at the LHC. In particular, with an integrated luminosity of $300 \mathrm{fb}^{-1}$ at the LHC, it is possible to probe resonance masses up to around $2 \mathrm{TeV}$.

Keywords: Top Physics; Electroweak Interaction; Beyond SM.
\end{abstract}

If there is no light Higgs boson found in the next generation of collider experiments, the interactions among the longitudinal vector bosons would become strong at a scale of $\mathcal{O}(1 \mathrm{TeV})$ and new dynamics must set in. The fact that the top-quark mass is very close to the electroweak scale $\left(m_{t} \approx v / \sqrt{2}\right)$ is rather suggestive: there may be a common origin for electroweak symmetry breaking and top-quark mass generation. A major goal for the Fermilab Tevatron and the CERN LHC is the detailed study of the properties of the top quark. In particular they should establish whether the third family behaves like the first two, or whether it is subject to new interactions.

This paper is a brief summary of a study reported elsewhere 1 , in which we introduce new resonances which couple strongly to the third generation but not necessarily to the $W$ and $Z$ gauge bosons. We are interested in new interactions of $b$ and $t$ quarks with these new heavy resonances. Our goal in this paper is to investigate the extent to which hadron colliders are sensitive to new interactions of the top quark regardless of the origin of the new interactions.

We begin with new vector resonance. We assume that this vector resonance has

*than@pheno.physics.wisc.edu

†valencia@iastate.edu

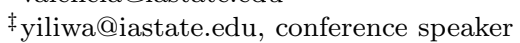


negligible couplings to electroweak gauge bosons. We thus proceed with the following effective Lagrangian coupling a spin one field to the top and bottom quarks,

$$
\mathcal{L}=-\Psi \gamma^{\mu}\left(g_{V}+g_{A} \gamma_{5}\right) \tau_{i} \Psi V_{\mu}^{i}
$$

In hadron colliders, however, light quark annihilation represents a significant production source for new vector resonances even if they couple predominantly to $b$ and $t$. To keep our study as model-independent as possible we choose a frame that make the contributions of the light quark annihilation small. For definiteness we will use the $Z^{\prime}$ model of Ref. ${ }^{2}$ in the limit of no $V-Z$ mixing.

$$
\begin{aligned}
\mathcal{L} & =\frac{g}{2} \tan \theta_{W} \tan \theta_{R}\left(\frac{1}{3} \bar{q}_{L} \gamma^{\mu} q_{L}+\frac{4}{3} \bar{u}_{R i} \gamma^{\mu} u_{R i}-\frac{2}{3} \bar{d}_{R i} \gamma^{\mu} d_{R i}\right) V_{\mu} \\
& -\frac{g}{2} \tan \theta_{W}\left(\tan \theta_{R}+\cot \theta_{R}\right)\left(\bar{t}_{R} \gamma^{\mu} t_{R}-\bar{b}_{R} \gamma^{\mu} b_{R}\right) V_{\mu} .
\end{aligned}
$$

In this expression $\theta_{R}$ is a new parameter. With large $\cot \theta_{R}$, this model provides a specific example of a new vector resonance which couples to $b$ and $t$ significantly and couples to the light fermions weakly. In the limit of large $\cot \theta_{R}$ these couplings are purely right-handed, with $g_{A}=g_{V}=(g / 4) \tan \theta_{W} \cot \theta_{R}$, in which $\cot \theta_{R}$ must be smaller than 20 if the new interaction remains perturbative.

We next consider a new scalar resonance. In this case we use a very simple (non-renormalizable) parametrization for the new interactions, and assume that the couplings of the scalar to the light fermions are completely negligible. We write

$$
\mathcal{L}=-\frac{m_{t}}{v} S\left(\kappa_{b} \bar{b} b+\kappa_{t} \bar{t} t\right) .
$$

This form allows us to parameterize simultaneously the cases where either the $b$ quark or the $t$-quark or both have enhanced couplings to the new scalar resonance. The tightest constraint is obtained by requiring perturbative unitarity 3 in the scattering amplitude $b \bar{b} \rightarrow b \bar{b}$ (or in $t \bar{t} \rightarrow t \bar{t}$ ) through an exchange of the new scalar. This leads to $\kappa_{b, t} \leq 3$.

we compute the contributions of the new interactions to multiple processes and conclude that Tevatron is not sensitive to this type of new physics. We find that processes with four heavy quarks, originating in the production of the heavy resonance in association with two heavy quarks are the most promising channels for the signal searchs in LHC. In the 4-quark process $p p \rightarrow b \bar{b} t \bar{t} X$ or $t \bar{t} t \bar{t} X$, the signal is completely dominated by the gluon fusion. There is also a much smaller contribution initiated by $b \bar{b}$ annihilation that we have calculated but not included. We use COMPHEP 4 to compute the signal and MADGRAPH 5 to compute the corresponding SM background. We implement cuts

$p_{T}(b)>100 \mathrm{GeV}, \quad p_{T}(t)>50 \mathrm{GeV}, \quad\left|y_{b}\right|<2, \quad M_{R}-4 \Gamma_{R}<m_{b \bar{b}, t \bar{t}}<M_{R}+4 \Gamma_{R}$

We present the sensitivity at the LHC assuming a total integrated luminosity of $300 \mathrm{fb}^{-1}$ in Fig. [1 With $50 \%$ of $b \bar{b}$-tagging efficiency and $0.5 \%$ faked b rate $\frac{6}{6}$, we end up a combined event efficiency of about $16 \%$ for $t \bar{t}$. We see that a $5 \sigma$ sensitivity may be reached for masses up to $2 \mathrm{TeV}$ for the vector resonance. 

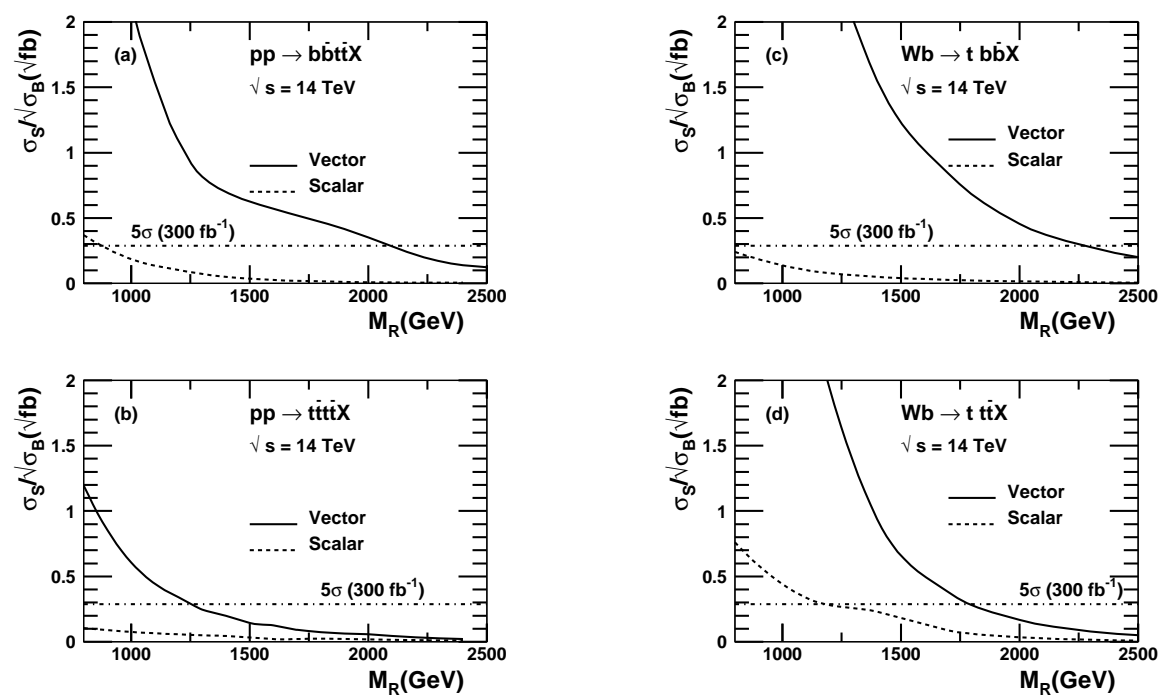

Fig. 1. Statistical sensitivity at the LHC with an integrated luminosity of $300 \mathrm{fb}^{-1}$ to a new resonance as a function of the resonance mass for (a) $p p \rightarrow b \bar{b} t \bar{t} X$, (b) $p p \rightarrow t \bar{t} t \bar{t} X$, (c) $w \rightarrow t, b \bar{b}$, and (d) $W \rightarrow t, t \bar{t}$.

It is well known that single top quark production via the electroweak process $W b \rightarrow t$ can be sizable due to the enhanced longitudinal gauge boson coupling at high energies. The cross section for single top production increases with energy up to about one-third of the cross section for $t \bar{t}$ pair production ${ }^{[7}$. The main advantage of this channel is the substantially smaller standard model background. We consider the $W b \rightarrow t$ process, associated with a heavy resonance that radiates off the top quark and decays to $b \bar{b}$ or $t \bar{t}$. We use cuts: $p_{T}(t, b)>100 \mathrm{GeV}$ and $\left|y_{t, b}\right|<2$. The high $p_{T}$ cut is imposed on all heavy quarks, including the two b quarks that reconstruct the resonance mass as well as the single top quark. Using the same $b$ and $t$ efficiencies as before we show in Fig. 1 the sensitivity in this case. The reach can be up to $M_{V} \sim 2 \mathrm{TeV}$ with a $5 \sigma$ significance.

\section{Acknowledgments}

The work of T.H. was supported in part by the US DOE under contract No. DEFG02-95ER40896, in part by the Wisconsin Alumni Research Foundation, and in part by National Natural Science Foundation of China. The work of G.V. and Y. W. was supported in part by DOE under contact number DE-FG02-01ER41155.

\section{References}

1. T. Han, G. Valencia and Y. Wang, Phys. Rev. D 70, 034002 (2004).

2. X. G. He and G. Valencia, Phys. Rev. D 66, 013004 (2002) [Erratum-ibid. D 66, 079901 (2002)]; X. G. He and G. Valencia, Phys. Rev. D 68, 033011 (2003); X. G. He and G. Valencia, arXiv:hep-ph/0404229 
4 T. Han, G. Valencia and Yili Wang

3. T. Han, Y. J. Kim, A. Likhoded and G. Valencia, Nucl. Phys. B593, 415 (2001);

T. Han, D. Rainwater and G. Valencia, Phys. Rev. D 68, 015003 (2003).

4. A. Pukhov et al., arXiv:hep-ph/9908288

5. T. Stelzer and W. F. Long, Comput. Phys. Commun. 81, 357 (1994).

6. Elizaveta Chabalina, private communication.

7. T. Stelzer, Z. Sullivan and S. Willenbrock, Phys. Rev. D58, 94021(1998); M. Beneke et al., arXiv:hep-ph/0003033 